\title{
Use of botulinum toxin-A for musculoskeletal pain in patients with whiplash associated disorders [ISRCTN68653575] Francisco J Juan*
}

\author{
Address: Department of Physical Medicine and Rehabilitation. Povisa Medical Center. C/ Salamanca, 5. 36211 Vigo, Pontevedra. Spain \\ Email: Francisco J Juan* - j.juan@arrakis.es \\ * Corresponding author
}

Published: I 3 February 2004

BMC Musculoskeletal Disorders 2004, 5:5
Received: 06 September 2003

Accepted: 13 February 2004

This article is available from: http://www.biomedcentral.com/147I-2474/5/5

(C) 2004 Juan; licensee BioMed Central Ltd. This is an Open Access article: verbatim copying and redistribution of this article are permitted in all media for any purpose, provided this notice is preserved along with the article's original URL.

\begin{abstract}
Background: Whiplash associated disorder is commonly linked to motor vehicle accidents and sports injuries. Cervical injury is attributed to rapid extension followed by neck flexion. The exact pathophysiology of whiplash is uncertain but probably involves some degree of aberrant muscle spasms and may produce a wide range of symptoms. The most commonly prescribed pharmacological agents for initial treatment of whiplash-associated pain are oral muscle relaxants and nonsteroidal anti-inflammatory drugs. However, potential systemic adverse effects limit these agents. Physical interventions such as mobilization, manipulation, and exercises have proved beneficial for pain and dysfunction but only on a time-limited basis. Little evidence suggests that physical therapy specifically aimed at the musculature (e.g., transcutaneous electrical nerve stimulation, ultrasonography, heat, ice, and acupuncture) improves prognosis in acute whiplash associated disorder. A new approach to treatment is the use of botulinum toxin, which acts to reduce muscle spasms.
\end{abstract}

Methods/design: This is a prospective, randomized, controlled clinical trial and botulinum toxinA $\left(\right.$ Botox $\left.{ }^{\circledR}\right)$ injections will be compared with placebo injections. The primary objective is to determine the efficacy of Botox ${ }^{\circledR}$ in the management of musculoskeletal pain in whiplash associated disorders.

Discussion: Botulinum toxin type-A toxin has been studied in small trials on whiplash associated disorder patients and has generally been found to relieve pain and improve range of motion. Specifically, we seek to assess the efficacy of Botox ${ }^{\circledR}$ in reducing pain and to improve the cervical spine range of movement, during the 6-month trial period.

\section{Background}

The term "whiplash" has been used to describe a mechanism of injury, and the various clinical manifestations as a consequence of the injury. Moreover, signs and symptoms have been designated the 'whiplash syndrome'. In 1995, the Quebec Task Force (QTF) on Whiplash Associated Disorders (WAD) adopted the following definition of whiplash: "whiplash is an acceleration-deceleration mechanism of energy transfer to the neck. It may result from rear-end or side-impact motor vehicle collisions, but can also occur during diving or other mishaps. The impact may result in bony or soft-tissue injuries (whiplashinjury), which in turn may lead to a variety of clinical manifestations called Whiplash Associated Disorders"[1]. Whiplash patients can be classified according to severity of signs and symptoms. The QTF-WAD classification sys- 
tem consists of five levels: WAD 0 indicates no complaints or physical signs; WAD I indicates neck complaints but no physical signs; WAD II indicates neck complaints and musculoskeletal signs (such as a decreased range of motion or muscle tenderness); WAD III and IV indicate neck complaints and neurological signs (WAD III) or fracture/dislocation (WAD IV), respectively. Research [2-5] has revealed that the most common presenting symptoms following an acute motor vehicle collision were neck pain (88-100 percent) and headache (54-66 percent). Other symptoms were neck stiffness, shoulder pain, arm pain/ numbness, paraesthesia, weakness, dysphagia, visual and auditory symptoms and dizziness.

The incidence of whiplash injury varies greatly throughout the world with rates between 70-200 per 100,000 inhabitants $[1,6]$. There is no consensus about the natural course of the whiplash injuries $[7,8]$. These authors concluded that between 14 and 42 percent of the whiplash patients developed chronic complaints (over six month duration), and that 10 percent of those patients had constant severe pain.

In most cases, WAD resolves quickly; $47 \%$ of injured people returns to normal activities within 4 weeks and only $2 \%$ continue to be absent from pre-accident activities 1 year after injury [1]. WAD is considered chronic if preaccident activity levels are not gained within 6 months. Ongoing cervical pain and reduced range of motion (ROM) associated with chronic WAD constitute both a prognostic and a therapeutic dilemma. Researchers have argued about whether chronic WAD even exists, suggesting that it might be a function of social conditioning, psychological makeup, and/or medico-legal compensation [9-14]. Despite this uncertainty, chronic neck pain does exist, and it can be initiated or perpetuated by an event such as a whiplash injury. The location and severity of tissue injury and the prognosis of chronic neck pain may depend on predisposing neck pathology. In addition to peripheral input, long-standing neck pain (like other chronic pain conditions) may be associated with a central component that can modify the pain threshold [15]. Considerable research has been dedicated to determine of the direct and indirect roles of the zygapophyseal joints in the generation of pain and dysfunction in whiplash injury [16]. Blocking the sensory nerves that innervate these joints reduced symptoms in $50 \%$ of the subjects with chronic whiplash pain [17]. However, this finding suggests that no responders might have been suffering from a pathology related not to the joints but to soft tissue. Almost nine of 10 whiplash sufferers had some degree of muscle spasm [18]. This aberrant muscle activity is further supported by use of surface electromyography (SEMG) in patients with whiplash revealed cervical muscle dysfunction [19]. In particular, these patients had decreased abil- ity to relax the trapezius muscles. Such a finding raises the question of whether cervical muscular dysfunction causes ongoing excess loading of the zygapophyseal joints, yielding the clinical picture of chronic whiplash, or whether muscle dysfunction is an attempt by the body to splint a subtly injured cervical spine.

The most commonly prescribed pharmacological agents for initial treatment of whiplash-associated pain are oral muscle relaxants and nonsteroidal anti-inflammatory drugs $[20,21]$. However, the effectiveness of these drugs in some patients is limited by their systemic therapeutic effect and unfavourable side effects. Physical interventions such as mobilization, manipulation, and exercises have proved beneficial for pain and dysfunction but only on a time-limited basis. Little evidence suggests that physical therapy specifically aimed at the musculature (e.g., transcutaneous electrical nerve stimulation, ultrasonography, heat, ice, and acupuncture) improves prognosis in acute WAD [8]. In chronic WAD, the only treatment that has clearly shown benefit is radiofrequency neurotomy [22]. Because only $49 \%$ of patients with whiplash have symptomatology related to cervical zygapophyseal joint pain, the remainder requires an alternate therapy [17].

The use of botulinum toxin (BTX) for the treatment of muscle pain in WAD has not been extensively studied or reported. This is also the case for chronic neck pain unassociated with a whiplash injury. Recently, a study exploring the potential benefits of relaxing selected neck muscles with botulinum toxin type-A (BTX-A) has been published [23]. In this randomized, placebo controlled trial, 28 subjects with chronic grade II WAD received injections of 100 units (U) of BTX-A or saline placebo. Each subject received bilaterally five injections of $0.2 \mathrm{ml}$ each into one or more of the following sites: splenius capitis, rectus capitis, semispinalis capitis, and trapezius. The five injection sites were chosen by palpation and corresponded to the five tenderest cervical muscular points. BTX-A was injected using a 30-gauge needle without electromyography (EMG) guidance. Follow-up assessments were carried out at 2 and 4 weeks after injection. Three outcome measures were considered: subjective pain, objective ROM in the neck, and subjective function. Subjective pain assessment was based on visual analogical scale (VAS) for neck pain, headache, and shoulder pain. At week 4 after injection, this group had significantly improved from pre-injection levels $(P<0.01)$. The placebo group did not demonstrate any significant changes at any time after treatment. This study is small and of short duration, but the results suggest that relaxing the cervical musculature is important in relieving pain and improving ROM in patients with WAD [24]. I have reported a significant reduction in pain as well as an improvement in ROM in $77.4 \%$ of patients responding significantly to a BTX-A injection in an open label 
study with 31 patients grade II WAD. Patients received 50-75 units of BTX-A, and the follow-up assessment was at week 8 [25]. BTX-A, specifically Botox ${ }^{\circledR}$, has been studied for the treatment of WAD and chronic neck pain. BTX type-B has been studied in patients with post-whiplash headaches [26].

\section{Methods/Design}

This is a randomized, prospective, double blind, placebocontrolled trial comparing BTX-A (Botox ${ }^{\circledR}$ ) with placebo. The study evaluates the efficacy and safety of Botox ${ }^{\circledast}$ in patients with musculoskeletal pain after whiplash associated disorders. The study will be conducted following all applicable institutional review board requirements and the subjects will provide written, informed consent before enrolment.

The trial will be at Whiplash Clinic in Povisa Medical Center, a tertiary referral center that sees approximately 800 patients per year with whiplash associated disorders. Patients will be referred from departmental and institutional colleagues as well as from regionally traffic accident insurances physicians.

\section{Randomization}

Patients eligible for inclusion will be randomized, and the study treatment started. Participants will be randomized by a computer-generated randomisation scheme and the clinical investigators evaluating each subject during the follow-up assessment will be blinded. The study computer will then randomly assign a treatment pack number that will identify one of the treatment packs stored in the department. Group A will receive treatment with Botox ${ }^{\circledR}$ and Group B will receive treatment with placebo injections. Documentation of treatment injection sites and volume injected will be recorded in the treatment procedure forms.

The treatment allocation schedule will be stored by the site's pharmacy. Study drug will be prepared by the pharmacy at each site. The study drug packages will be labeled with the identification number from the treatment allocation schedule.

\section{Blinding}

Both the patient and principal investigator and his staff will be blinded as to the identity of the randomized study medication. Blinding will be maintained by having a designated pharmacist (the only person to have access to the randomization list) providing the principal investigator or study coordinator with a vial containing the study medication labeled with the patient's sequential identification number from the randomized allocation schedule. All patients will remain double blind until after the last patient has completed the study. Patients in each group will complete an 6-month follow-up.

\section{Inclusion criteria}

- Patients with grade II WAD of QTF-WAD

- Neck pain secondary to cervical whiplash injury with muscoskeletal signs* of $>3$ months.

$\bullet>18$ years.

- Lack of response to conventional physical and medical therapy

\section{${ }^{*}$ Muscoskeletal signs}

- Palpable band, spot tenderness, and jump sign in cervical muscles or restricted range of motion in cervical spine.

- Demonstrated precipitation of neck pain with external pressure over the occipital or cervical region on affected side.

- Myofascial pain of cervical muscles.

- Experienced pain on maneuver of stretching,

- Trigger point with associated referred pain.

\section{Exclusion criteria}

- Serious somatic or psychiatric illness

- Anticoagulation therapy

- Myasthenia gravis

- Pregnancy or breast-feeding

- Abnormal anatomy

- Rheumatoid disease or radiculopathy, need for regular analgesic for severe pain

- Fixed contracture

\section{Patient Screening}

Prospective patients will be subjected to a preliminary examination, which will include general medical history, and complete physical and neurological examination. Those meeting the provisional eligibility requirements of the study will be asked to sign the Patient Consent Form.

All patients who are given the screening examination will have their name entered into a Screening/Enrollment log along with the date and time of examination. If the 
patient is ineligible for participation, an explanatory note will be provided in the Screening/Enrolment Log. Randomization numbers will only be assigned to patients who are fully eligible, and agree to participate in the study.

\section{Study treatment}

- Botox ${ }^{\circledast}$ (Botulinum Toxin Type-A, Allergan, Inc., Irvine, CA) is supplied as a sterile, preservative-free, vacuum dried powder consisting of 100 units of Clostridium botulinum toxin, $0.5 \mathrm{mg}$ human albumin, and $0.9 \mathrm{mg}$ of sodium chloride. Unpreserved sterile $0.9 \%$ saline solution is used for resolubilization of the Botox ${ }^{\oplus}$ powder, and will be used to prepare a $100 \mathrm{unit} / \mathrm{ml}^{\text {Botox }}{ }^{\circledast}$ solution.

\section{Clinical use and safety of Botox ${ }^{\circledR}$}

We use Botox ${ }^{\otimes}$ and this protocol cannot be used to administer any other commercial preparation of BTX. Botox ${ }^{\circledast}$ is a neurotoxin, is effective for treating a variety of disorders of involuntary muscle contraction, including spasticity, cervical dystonia, blepharospasm and hemifacial spasm. It inhibits neuromuscular signaling by blocking the release of acetylcholine at the neuromuscular junction. The biological effects of the toxin are transient with normal neuronal signaling returning within approximately 3-6 months post injections. Botox ${ }^{\oplus}$ is available in a standard vial that contains 100 units of toxin. The vacuum dried toxin is reconstituted with 0.9 percent unpreserved sterile saline to various concentrations just before usage, depending on the indication. Preservatives in the saline could inactive the toxin. The injection of the diluents into the vial must be performed according to manufacturer's recommendations with gentle mixing after allowing the vacuum in the vial to draw in the injected diluents.

- Saline Solution: Unpreserved, sterile $0.9 \%$ saline solution will be obtained from the following commercial source: $\mathrm{B} /$ Braun $10 \mathrm{ml}$ sterile saline solution.

- Placebo: The placebo will consist of the saline solution described in previous section.

\section{Treatment}

Up to a total of $1 \mathrm{ml}$ of $0.9 \%$ saline (placebo treatment) or active treatment (Botox ${ }^{\circledR}$ ) will be injected; the following muscles will be injected in all patients: splenius capitis, semiespinalis, and trapezius. Each subject will receive five injections of $0.2 \mathrm{ml}$ (20 units) each into one or more of the following sites: splenius capitis, semispinalis capitis, and trapezius. The five injection sites will be chosen by palpation and corresponded to the five tenderest cervical muscular points. The number of injection sites per muscle will be: trapezius three sites, semiespinalis one site, and splenius one site. Maximum volume per site will be of 0.2 $\mathrm{ml}$ (20 units of $0.9 \%$ saline or active treatment). Total maximum dose per treatment of Botox ${ }^{\circledast}$ will be of 100 units.

The concentration of this $0.9 \%$ saline solution reconstituted Botox ${ }^{\circledast}$ will be 100 units $/ \mathrm{ml}$, which means that the amount of active treatment to be injected into the various muscle groups will range from 20 to 60 units. The study investigators will administer treatment using a combination of fixed injections sites and injection at sites of pain or tenderness.

\section{Injection technique}

The injection technique used for the neck is based on experience with cervical dystonia. The sites chosen are chiefly in the large superficial muscles, specifically, the splenius capitis, rectus capitis, semispinalis capitis, and trapezius. These muscles can easily be palpated in most individuals and can be injected without sophisticated techniques. Most patients exhibit tender areas in the larger muscles, often in conjunction with tight bands or knots. These tender areas do not always meet the definition of classic trigger points.

In our centre, Botox ${ }^{\circledast}$ is typically diluted to $10 \mathrm{U}$ per $0.1 \mathrm{ml}$ of saline. In the large muscles of the neck and back, relatively large volumes can be injected without diffusion to adjacent structures. In the face or anterior neck, these larger volumes are more likely to lead to unwanted paralysis of neighbouring muscles [27]. The optimal dilutions have not yet been determined.

During the injection technique, we do not use EMG guidance or muscle stimulation.

To determine if the dose has been insufficient because of excessive toxin distribution, large muscle bulk, or possibly antibodies, providers can inject a small cosmetic dose into the frontals muscle to verify the patient's response to the toxin.

\section{Adverse events}

An adverse event is defined as any medical occurrence whether or not related to treatment (including worsening of a preexisting medical condition). They will be recorded on the case report form provided for that purpose. If an adverse event should occur, treatment will be provided by the principal investigator or co-investigators, and followup will continue until the event resolves. The principal or co-investigators will also be responsible for characterizing the nature and severity of the adverse event, its relationship to the study medication (unrelated, uncertain, possible, and probable), any action taken to remedy the event, and patient outcome. An adverse event that is serious, irrespective of its relationship to treatment, will be recorded and reported appropriately. 


\section{Precautions}

BTX-A treatment is potentially a painful procedure. It is contraindicated in the presence of infection at the injection site(s) and in individuals with known hypersensitivity to any ingredient in the formulation.

Individuals with peripheral motor neuropathic diseases or neuromuscular functional disorders should receive BTX-A treatment with caution. BTX-A should also be used with caution in patients receiving aminoglycosides or other agents interfering with neuromuscular transmission.

Formation of neutralizing antibodies to BTX-A may reduce its effectiveness by inactivating the biological activity of the toxin. The rate of formation of these neutralizing antibodies in patients receiving BTX-A treatment has not been well studied and the critical factors for neutralizing antibody formation have not been well characterized. The effect of the long-term use of BTX-A has not been studied, however, the reformulated BTX-A has a lower protein content that may decrease the risk of antibody formation and the development of resistance.

\section{Study phases}

There is a 6-month treatment and follow-up phase. There will be a total of 12 clinic visits during the study period. The first visit will be the patient's baseline evaluation, randomization and treatment visit, and follow-up assessment will be performed at 15 days intervals. Patients will return to the Whiplash Clinic at 15 days, intervals, and all assessment scales will be recorded at each follow-up visit. At the final visit, a patient discontinuation form will be completed for each patient. If a patient discontinues prematurely, the outcome measures will be obtained at the time of discontinuation.

\section{Outcomes measures}

Outcome measures include ROM, subjective pain and disability measure via VAS and Neck Pain Disability Index (NDI). Objective tenderness to palpation will be recorded using previous scales and systems.

\section{Assessment}

Objective assessment of treatment outcome of whiplash can be difficult. Since 80 percent of patients responded in the open-label trial, the success rate of the controlled study should be downward adjusted by 20 percent. Comparison between studies is difficult, due to variations in dosages, treatment protocols, evaluation methods, injection technique (single versus multiple sites), rating scales, and definitions of improvements.

The following variables will be measured:

\section{Subjective pain}

Assessment using visual analogue scales (VAS) to assess most severe pain and average pain intensity during the study. VAS is a $10 \mathrm{~cm}$ pain intensity scale. In this numerical rating scale $(0=$ no pain, $10=$ unbearable pain o the worst pain experienced). The total score of three VAS assessments will be reported.

\section{Assessment of disability}

Assessment of disability due neck pain with the NDI, a modified version of the Oswestry Low Back Pain. [28].

\section{Assessment of range of neck motion}

We use $\mathrm{CROM}^{\circledR}$ Cervical Range of Motion Instrument, with measurements of rotation, flexion, extension, and lateral bending. Assessment of cervical Range of Motion by $\mathrm{CROM}^{\circledast}$ : It is a magnetic inclinometer device. The CROM $^{\circledast}$ Cervical Range of Motion Instrument provides accurate, repeatable measurements of cervical movement and objective data that clinical studies required. The system combines inclinometers and magnets to provide accurate measurements of cervical range of motion in an easy-to-use instrument. It eliminates positioning, zeroing and tracking errors common with stand-alone inclinometers. It allows rotation movements in upright position. Standard protocol minimizes chance for examiner's errors.

\section{Assessment of health status}

We use a generic measure of health status with the medical outcomes study 36-item short-form health survey (SF36) [29].

\section{Tenderness to palpation}

Reaction to pressure will be graded from 0 to 3 with respect to discomfort $(0=$ no discomfort, $1=$ minimal, $2=$ moderate, $3=$ severe discomfort with minimal pressure); this classification was used in previous studies.

In addition, we will use the Mechanical Algometer Pain Test ${ }^{\circledast}$ from Wagner Instruments, USA. Many clinical applications of the Algometer have been documented, including evaluation and identification of trigger points [30], evaluation of pain sensitivity [31,32], Pressure measurement has also been shown effective for evaluating the results of pain relieving modalities such as anaesthetic blocks, heat, manipulation, anti-inflammatory, as well as documenting long-term treatment effectiveness [31,33]. Because of its reliability and reproducibility, algometry can be used for objective medico-legal documentation of pain intensity [34].

The methodology for assessment of palpation tenderness using the Algometer Pain Test ${ }^{\circledR}$ is: a) Patient lie down, exposing the areas to be tested. Supporting pillows should 
be used to assist the patient in relaxing the muscles overlying the tender points. b) Explain the procedure to the patient. Have the patient indicate the onset of pain with a verbal cue such as "Yes" or "Stop", or by squeezing the "Enter" footswitch. This adds another level of examiner objectivity to the test. c) Use one of the standard test points, or has the patient point to the specific area of discomfort. Palpate with your fingertip to identify the point of maximum sensitivity. Mark that point with a pen and document its location with reference to an anatomical landmark for future testing. d) Locate, mark, and document the same point on the contralateral side if appropriated. e) Use the appropriate tip for the area of the body being tested. Typically, the $1.0 \mathrm{~cm}^{2}$ tip for torso and extremities, and the $0.5 \mathrm{~cm}^{2}$ for the head and neck work best. Denote the tip size in the dialog. f) Remind the patient to stop the test upon the onset of pain, and place the applicator tip over the mark. Apply force perpendicular to the skin's surface at a gradual rate of 2.2 pounds ( 1 $\mathrm{kg}$ ) per second. Remove the Algometer when the patient stops the test. Multiple readings may be taken to improve reliability. g) Test the contralateral side if not a midline test.

Pressure Tolerance Measurement: a) Have the patient lie down, exposing the middle deltoid and the shin. b) Palpate the bulk of the deltoid muscle to rule out the presence of local tenderness and/or pathology. Mark the point of maximum muscle bulk with a pen. c) Explain the procedure to the patient. Have the patient indicate when the pain becomes too great with a verbal cue or by squeezing the "Enter" footswitch. d) Place the unit with the $1 \mathrm{~cm}^{2}$ applicator tip over the mark. Apply force perpendicular to the skin's surface at a gradual rate of 2.2 pounds per second. Remove the Algometer when the patient stops the test. e) Multiple readings on the same site or contralateral side may be performed to improve measurement reliability. f) Repeat the procedure using the mid-tibia.

\section{Surface electromyography}

In order to find excessive electrical activity of muscles during movement, hyperactivity after contraction and/or inappropriate co-activation. SEMG activity of the upper trapezius muscles is bipolar, recorded according the recommendations of SENIAM [35].

The amplitude of the SEMG using MYOMED ${ }^{\circledR} 932$ (EnrafNonius, Inc.) signals is usually expressed as some number of microvolts, noted as series of relatively instantaneous measurements, or averaged or integrated over a clinically meaningful period. Amplitude analyses are conducted to evaluate the magnitude and timing pattern of muscle activity. Use of SEMG amplitudes has been described for examination and feedback training in a wide variety of musculoskeletal disorders $[36,37]$. Clinically less com- mon than amplitude analyses, investigation in the frequency domain is performed to study muscular fatigue. SEMG amplitude, tracings, may be processed to, reveal a range of component frequencies, the spectrum of which shifts in a reliable way with fatigue [38]. That is, the frequency spectrum becomes compressed toward slower values due to neuromuscular and metabolic changes associated with high intensity isometric contractions. The shift begins as the contractions are sustained beyond a short time, preceding the actual loss of force, and continues as force declines. This way of fatigue monitoring may have certain advantages over other measures [39] and successfully discriminates spinal pain patients from control subjects with impressive accuracy $[40,41]$.

Several theories about musculoskeletal pain syndromes such as WAD suggest that pain and muscle activity interact and may contribute to the chronicity of symptoms. Studies using SEMG have demonstrated abnormal muscle activation patterns of the upper trapezius muscles in the chronic stage of WAD (Grade II) [19]. The acute stage is characterized by a reorganization of the muscular activation of neck and shoulder muscles, possibly aimed at minimizing the use of painful muscles [42]. Studies with SEMG show excessive electrical activity of muscles during movement, hyperactivity contraction, and/or inappropiate coactivation with other muscles during movement.

The following protocol will be use in WAD using SEMG: Skin adequately prepared, shaved and cleaned with $70 \%$ alcohol. SEMG activity of the upper trapezius muscles recorded bipolar, amplified using MYOMED ${ }^{\circledR}$ 932. Electrode placements, according SENIAM. Patients seated in upright position. $\mathrm{Ag} / \mathrm{AgCl}$ pregelled electrodes with a circular diameter of $10 \mathrm{~mm}$. Inter-electrode distance of \pm 20 $\mathrm{mm}$. The reference electrode over the spinous process of $\mathrm{C} 7$, and electrode $2 \mathrm{~cm}$ laterally to the midpoint of the line between the acromion and the spinous process of vertebra C7. After electrode placement they are fixed to the skin with tape. We recorded the muscle activity during four stages:

- Baseline: Back supported, the hips and knees at $90^{\circ}$ and the hands resting in the lap.

- Reference electrical activation: while the arms are hold straight and horizontally at $90^{\circ}$ abduction in the frontal plane of the body with the hand relaxed, and palms pointing downward.

- Physical exercises: The patient is asked to move his or her dominant arm between three target areas by making circles with a diameter of $70 \mathrm{~mm}$ using a pencil. Rest of the non-dominant arm. 
- Final or post-exercise: Immediately after exercise or abduction of the arms we obtained the muscles activity.

According to Nederhand et al [19] we use the muscle activation patterns of upper trapezius: time related recovery pattern, mean level of pre-exercise and mean level of postexercise. We record excessive electrical activity, inappropriate co-activation of others muscles during movement.

\section{Sample size}

Sample size calculation: Earlier studies have indicated that it would be possible to enrol 160 patients WAD II within 12 months in areas with 400,000 inhabitants.

Most sample size calculations involve estimating the number of observations needed to compare two means by using Student's t test for independent samples or two proportions by using Pearson's chi-square test. Standard practice is to determine the sample size that gives an $80 \%$ chance of rejecting the hypothesis of no difference at the 0.05 level of significance.

We have assumed a correlation between baseline measures and outcome scores of 0.4 , based in previous studies about randomized clinical trial design in whiplash associated disorders. [43]

A sample of 90 subjects per group provides at least an $80 \%$ probability of detecting an effect of botulinum toxin of $1.5 \mathrm{~cm}$ on a $10 \mathrm{~cm}$ VAS pain intensity measure and $10 \%$ on the $0-100 \%$ NPDI.

In addition, we assumed that the standard deviation (SD) of pain intensity is $2.5 \mathrm{~cm} \mathrm{[44-46]} \mathrm{and} \mathrm{the} \mathrm{SD} \mathrm{for} \mathrm{NPDI} \mathrm{is}$ $20 \%$ [47-49], and that alpha is 0.05 .

\section{Data analysis}

The primary outcome measure is the change from baseline at week 8 . The mean change in the Botox $\left({ }^{\circledR}\right)$ group will be compared to that of the placebo group, and the statistical significance will be calculated by using the twosample t test. Patients will be included in the primary analysis on the basis of intention-to-treat. Patients who withdraw prematurely will have week 8 outcomes measured at the time of withdrawal.

All analyses will be intention-to-treat analyses.

The analytic strategies will be as follows: ordinary least squares regression models for each continuous outcome from baseline to follow up assessment: logistic regression, to estimate the effects of treatment on dichotomous outcomes and mixed effects linear models, to identify changes by treatment group over the follow-up.
Age, sex, SF-36 mental score, baseline outcome value, duration of neck pain will be included in the ordinary least squares and logistic models. The mixed effects models include the same covariates except baseline outcome value. The logistic model includes results with odds ratios and $95 \%$ confidence intervals for each treatment contrast.

\section{Informed consent}

All patients will sign the appropriate institutional review board and ethics review committee approved protocol specific informed consent prior to any protocol procedures. The consent form will include all relevant information from the patient package insert of the product. Consent will be documented by patient's dated signature on a consent form along with the dated signatures of the person conducting the consent discussion.

\section{Efficacy}

BTX-A injections are not a curative treatment. BTX-A provides a temporary paralytic effect and requires repeated injections to continue the beneficial effects. The duration of effect is longer with the initial injection and progressively gets shorter with repeated injections for most dystonic disorders. It is not known at this time whether BTXA can be readministered indefinitely or if the effectiveness will wear off over time.

Primary efficacy measure: change in mean monthly pain and ROM during the $3^{\text {rd }}$ and $6^{\text {th }}$ study month compared to the pre-trial period and compared with the placebotreated group: medications requirements and improvement of symptoms compared with the placebo treated group. Finally, improved health-related disability compared with placebo-treated group.

\section{Discussion}

BTX-A (Botox ${ }^{\varpi}$ ) has been found to be safe and effective for a number of clinical conditions. Presently, BTX-A has been approved by the Federal Drug Administration. (FDA) for the treatment of cervical dystonia in adults to decrease the severity of abnormal head position and neck pain associated with cervical dystonia, and for treatment of strabismus and blepharospasm associated with dystonia.

Listed below are clinical applications for which BTX-A is not approved by the FDA, but where data exists to support the clinical use of BTX-A:

- Spasmodic torticollis

-Writer's cramp with significant wrist-joint deviation

- Spasmodic dysphonia 
- Dynamic contracture in cerebral palsy patients

- Post Stroke Spasticity

- Myofascial pain syndrome

- Chronic low back pain

Following are the clinical conditions where insufficient data exists to support the clinical use of BTX-A and none of these are FDA approved indications:

- "Off" painful dystonia in Parkinson's disease patients

- Rigidity in Parkinson's disease patients

- TMJ disorders and Bruxism

- Fixed contracture in cerebral palsy patients

- Spasticity associated with Multiple sclerosis

- Tension headache

- Migraine

- Whiplash associated with neck-pain

- Spasticity associated with Perinatal hypoxia

- Progressive supranuclear palsy

- Cortical basal degeneration

- Extensor hallucis longus dystonia

- Writer's cramp with neutral wrist position

- Essential tremor

- Stenographer's cramp

- Sixth nerve palsy

\section{Botulinum toxin}

The strains of Clostridium botulinum produce a group of the most potent biological toxins that act by preventing synaptic transmission of acetylcholine across the neuromuscular junction. BTX is classified into eight distinct serologically related neurotoxins, seven of which can cause paralysis. Three of these types A, B and E are also associated with epidemic botulism from food poisonings. The BTX are members of the same class of drugs, and as such, show important similarities and differences.
Although these toxins are antigenically distinct (i.e. different serotypes), they possess similar molecular weights, and have a similar subunit structure, though different amino acid sequences. The active toxins have a molecular weight of approximately 150,000 Daltons and are composed of a heavy chain $(100,000$ Daltons $)$ that is linked by a disulfide bond to a light chain (50,000 Daltons) associated with a single Zinc atom. BTX exerts its effect at the neuromuscular junction by inhibiting the release of acetylcholine, and this in turn causes flaccid paralysis. BTX-A causes chemical denervation by preventing synaptic transmission and effectively weakens the muscle. BTX-A injected into a muscle binds irreversibly to the nerve, where it is internalised into the nerve ending, causing paralysis of the muscle by preventing the release of acetylcholine. BTX-A binds rapidly and with high affinity to the nerve, however, its maximal paralytic effect peaks four to seven days after the injection. Very little toxin reaches the systemic circulation. Muscle paralysis is dose dependent and reversible. Recovery occurs either when new portions of the nerve, called axonal terminals, sprout and reinnervate the muscle or when the original terminal is reactivated. Side effects can occur when too much BTX-A is injected, when the drug is not contained in an injected muscle or, very rarely, when an underlying syndrome that is latent such as Myasthenia Gravis is unmasked by the injection. Clinical effects of BTX-A injections lasts from two to six months or more depending on the dose and condition being treated and repeated treatments are often necessary to control abnormal muscle functioning. Treatment with BTX-A does not result in a return to normal muscle functioning since the underlying cause is still present. BTX-A treatment weakens specific muscles, allowing graded movements, unlike other pharmacological treatments that weaken all muscles.

\section{Theoretical pharmacological rational for effect in whiplash associated disorder}

Neck pain after whiplash injuries is a common finding. Up to $87 \%$ of patients with WAD have some degree of muscle spasm. BTX has demonstrated efficacy in a variety of conditions associated with muscle spasms. Patients with WAD have pain-limiting range of motion in the neck and increased pain with function. In addition, these patients exhibit marked localized tenderness and often refer pain to distant sites like active myofascial trigger points (MTrPs). Exactly how botulinum toxin works has not been established, but it is presumed that it decreases active tender points. Unlike standard tender point injections, BTX produces long-term responses without significant adverse events. BTX success in pain management is attributed to its ability to block acetylcholine from being released at synapses. The neurotoxin effects are thought to act only upon motonerve endings while sensory nerve fibers are spared from such effects. Thus, analgesic effects are 
likely to occur, not as a result of blocking afferents sensory fibbers at the site of injection, but rather from secondary effects that may be attributed to muscle paralysis, improved blood flow, and release of fibers under compression by abnormally contracting muscles. The primary action may affect alpha and gamma motoneuron function in the muscle spindles resulting in lower muscle tone.

Although still under extensive study, BTX appears to have a role in the diagnosis and the treatment of chronic WAD. As a diagnostic tool, injection of BTX can identify those cases of whiplash disorder that are primarily myogenous in nature. As a therapeutic tool, BTX provides a safe, minimally invasive, reversible means of treating affected muscles.

\section{Human participation protection}

The study protocol and informed conset form will be approved by the institutional review board of the Povisa Medical Center and the Regional Commission for Clinical trails as well as the National Commission of Health Ministry.

\section{Competing interests}

Dr. Francisco. J. Juan is expert using BTX in neurological diseases and WAD, and imparts some training courses for Allergan, Inc. in Spain to physician interested in this field.

The company Allergan is the supplier of Botox ${ }^{\circledR}$ and won't be involved in any form in this study.

I have not received any financial support or benefits in any form from a commercial party related directly or indirectly to the subject of this study protocol.

I am grateful to people affiliated to this company the support in review the manuscript and make English corrections.

\section{References}

I. Spitzer WO, Skovron ML, Salmi LR, Cassidy DJ, Duranceau J, Suissa $S$, Zeiss E : Scientific monograph of the Quebec Task Force on whiplash-associated disorders: redefining "whiplash" and its management. Spine 1995, 20(8 SuppI): IS-73S.

2. Norris $\mathrm{SH}$, Watt I: The prognosis of neck injuries resulting from rear-end vehicle collisions. J Bone Joint Surg $\mathrm{Br} 1983$, 65:608-6II.

3. Hildingsson C, Toolanen G: Outcome after soft-tissue injury of the cervical spine. A prospective study of 93 car-accident victims. Acta Orthop Scand 1990, 6 I:357-359.

4. Radanov BP: Epidemiology of whiplash. Ann Rheum Dis 2000, 59:394-6. Letter.

5. Stovner LJ: The nosologic status of the whiplash syndrome: a critical review based on a methodological approach. Spine I 996, 2 I (23):2735-2746.

6. Miles KA, Maimaris C, Finlay D, Barnes MR: The incidence and prognostic significance of radiological abnormalities in soft tissue injuries to the cervical spine. Skeletal Radiol 1988, I 7:493-496.

7. Croft AC, Freeman MD: 'Railway spine' to 'late whiplash': cases of wrongful controversy? Top Clin Chiro I998, 5(3):54-6I.
8. Freeman MD, Croft AC, Rossignol AM: Whiplash associated disorders: refining whiplash and it's management' by the Quebec Task Force. Spine 1998, 23(9): I043-1049.

9. Obelieniene D, Schrader H, Bovim G, Miseviciene I, Sand T: Pain after whiplash: a prospective controlled inception cohort study. J Neurol Neurosurg Psychiatry 1999, 66:279-83.

10. Giebel GD, Bonk AD, Edelmann M, Huser R: Whiplash injury. J Rheumatol 1999, 26:1207-8. Letter.

II. Keidel M, Baume B, Lüdecke C, Freihoff J, Wilhelm H, Rieschke P, Vandenesch P, Diener HC: Prospective analysis of acute sequelae following whiplash injury. World Congress on Whiplash-Associated Disorders, Vancouver BC/Canada 1999, I 27:Abstr 45. February 7II, 1999

12. Partheni M, Miliaras G, Constantoyannis C, Papadakis N: Whiplash injury. J Rheumatol 1999, 26: I206-7. Letter

13. Ferrari R, Russell AS: Epidemiology of whiplash. Ann Rheum Dis 2000, 59:394-6. Letter.

14. Radanov BP: Epidemiology of whiplash. Ann Rheum Dis 2000, 59:394-6. Letter.

15. Curatolo M, Petersen Felix S, Arendt Nielsen L, Giani C, Zbinden AM, Radanov BP: Central hypersensitivity in chronic pain after whiplash injury. Clin J Pain 200I, I 7:306-I5.

16. Lord S, Barnsley L, Bogduk N: Cervical zygapophyseal joint pain in whiplash. Spine 1993, 7:355-71.

17. Lord SM, Barnsley L, Wallis BJ, Bogduk N: Chronic cervical zygapophyseal joint pain after whiplash: a placebo-controlled prevalence study. Spine 1996, 2 I:1737-45.

18. Wiley AM, Lloyd J, Evans JG: Musculoskeletal sequelae of whiplash injuries. Adv $Q$ 1986, 7:65-73.

19. Nederhand MJ, Hermens HJ, ljzerman MJ, Turk DC, Zilvold G: Cervical muscle dysfunction in the chronic whiplash associated disorder grade II (WAD-II). Spine 2000, 25: 1938-43.

20. Percy J: Whiplash syndromes: a doctor's dilemma. Can J CME 1994:63-7.

21. Sutherland R: A crash course in whiplash management. Patient Care 1993, 9:25-34.

22. McDonald GJ, Lord SM, Bogduk N: Long-term follow-up of patients treated with cervical radiofrequency neurotomy for chronic neck pain. Neurosurgery 1999, 45:6I-8.

23. Freund $B$, Schwartz $M$ : Treatment of whiplash associated neck pain with botulinum toxin A: a pilot study. J Rheumatol 2000, 27:48I-4.

24. Freund B, Schwartz M: Use of botulinum toxin in chronic whiplash associated disorder. The clinical Journal of Pain 2002, I 8(6 supplement):SI63-168.

25. Francisco J Juan: Treatment of neck pain after whiplash injuries with botulinum toxin-A injections. Journal of Musculoskeletal Research 2003, 7(3\&4):221-223.

26. Opida CL: Open-label study of Myobloc $^{\mathrm{TM}}$ (botulinum toxin type $B$ ) in the treatment of patients with post-whiplash headaches. Poster 204 presented at: International Conference 2002: Basic and Therapeutic Aspect of Botulinum and Tetanus Toxins; Hannover, Germany. 2002.

27. Caruthers A, Caruthers J: Cosmetic uses of botulinum A exotoxin. Adv Dermatol 1997, I 2:325.

28. Vernon H, Mior S: The neck disability Index: a study of reability and validity. J Manipulative Physiol Ther 199I, I 4:409-4I5.

29. Ware Je, Sherbourne CD: The MOS 36-Item Short-Form health Survey (SF-36). Med Care 1992, 30:473-483.

30. Fischer AA: Documentation of myofascial trigger points. Arch Phys Med Rehab 1988, 69:286-291.

31. Fischer AA: Pressure algometry over normal muscles. Standard values, validity, and reproducibility of pressure threshold. Pain 1987, 30: I I5-126.

32. Keele KD: Pain sensitivity tests: pressure algometer. Lancet 1954, 1:636-639.

33. Merskey H, Spear FG: Reliability of pressure algometer. Br J Soc Clin Psychol 1964, 3:130-136.

34. Fischer AA: Pressure Threshold Meter: Its use for quantification of tender spots. Arch Phys Med Rehab 1986, 67:836-838.

35. Hermens HJ, Freriks B, Merletti R, Hagg G, Stegeman D, Blok J, Rau GC: European Recommendations for surface electromyography. Roessing Research and Development Enschede 1999. ISBN 9075452-15-2

36. Cram JR, Kasman GS: Introduction to Surface Electromyography. Gaithersburg, MD: Aspen Publishers; 1998. 
37. Kasman GS, Cram JR, Wolf SL: Clinical Applications in Surface Electromyography: Chronic Musculoskeletal Pain. Gaithersburg, MD: Aspen Publishers; 1998.

38. Basmajian JV, De Luca CJ: Muscles Alive: Their Functions Revealed by Electromyography,. 5th edition. Baltimore, MD: Williams \& Wilkins; 1985.

39. $\mathrm{Ng} J \mathrm{KF}$, Richardson CA, Jull GA: Electromyographic amplitude and frequency changes in the iliocostalis and multifidus muscles during a trunk holding test. Phys Ther 1997, 77:954-96I.

40. Roy SH, Oddsson LI: Classification of paraspinal muscle impairments by surface electromyography. Phys Ther 1998, 78:838-85I.

41. Gogia P, Sabbahi M: Median frequency of the myoelectric signal in cervical paraspinal muscles. Arch Phys Med Rehabil 1990, 7I:408-4I4.

42. Nederhand MJ, Hermens HJ, IJzerman MJ, Turk DC, Zilvold G: Chronic neck pain disability due to an acute whiplash injur. Pain 2003, I02(I-2):63-7I.

43. Stewart M, Maher C, Refshauge K, Herbert R, Bogduk N, Nicholas M: Advice or exercise for chronic whiplash disorders? Design of a randomized controlled trial. BMC Musculoskeletal Disorders 2003, 4: 18

44. Westaway M, Stratford P, Binkley J: The patient-specific functional scale: validation of its use in persons with neck dysfunction. Journal of Orthopaedic and Sports Physical Therapy 1998 , 27:33I-338.

45. Lord S, Barnsley L, Wallis B, McDonald G, Bogduk N: Percutaneous radio-frequency neurotomy for chronic cervical zygapophyseal-joint pain. New England Journal of Medicine 1996, 335:172I-6.

46. Barnsley L, Lord S, Wallis B, Bogduk N: Lack of effect of intraarticular corticosteroids for chronic pain in the cervical zygapophyseal joints. New England Journal of Medicine 1994, 330: 1047-50.

47. Riddle $D$, Stratford $P$ : Use of generic versus region-specific functional status measures on patients with cervical spine disorders. Physical Therapy 1998, 78:95I-963.

48. Hains F, Waalen J, Mior S: Psychometric properties of the Neck Disability Index. Journal of Manipulative and Physiological Therapeutics 1998, 21:75-80.

49. Westaway M, Stratford P, Binkley J: The patient-specific functional scale: validation of its use in persons with neck dysfunction. Journal of Orthopaedic and Sports Physical Therapy 1998, 27:33I-338.

\section{Pre-publication history}

The pre-publication history for this paper can be accessed here:

http://www.biomedcentral.com/1471-2474/5/5/prepub
Publish with Bio Med Central and every scientist can read your work free of charge

"BioMed Central will be the most significant development for disseminating the results of biomedical research in our lifetime. "

Sir Paul Nurse, Cancer Research UK

Your research papers will be:

- available free of charge to the entire biomedical community

- peer reviewed and published immediately upon acceptance

- cited in PubMed and archived on PubMed Central

- yours - you keep the copyright
BioMedcentral 